\section{Diffraction of Light by Ultra-Sonic Waves}

IN a recent paper, $R$. Lucas $^{1}$ has described an experiment designed to test the relative merits of a theory, proposed by C. V. Raman and N. S. Nagendra Nath, of the scattering of light by high-frequency sound waves and that due to himself and P. Biquard. The two theories differ in that, while Raman and Nath assume only a change in phase of normally incident light as it progresses through the sound field, Lucas and Biquard's theory takes account of changes in both amplitude and phase. Lucas's experiment appears to prove by indirect methods that the theory of Raman and Nath is true only for sound fields of thicknesses of the order of $4 \mathrm{~mm}$. or less. R. Bär ${ }^{2}$ has also reported on an experiment which indicates that while the Raman-Nath theory predicts a type of intensity distribution which agrees qualitatively with the observed patterns at frequencies of 1.5 and 7.5 megacycles, there are several discrepancies which indicate that the fundamental assumption of these authors is incorrect. The portion of their theory dealing with oblique incidence apparently gives results which are not in accord with the observations, particularly at the higher frequency. Furthermore, Bär has obtained 'photographs' of the actual ultra-sonic grating by the insertion of a photographic plate in an appropriate position in the light beam behind the stationary sound field. With no lens system of any type to focus the light rays, a series of quite sharp fringes appears on the plate, indicating the presence of some changes in light amplitude as well as in phase.

I have recently completed some experiments, which are to be published shortly in the Canadian Journal of Research, in which the distribution of light energy among the various diffraction orders has been measured as a function of ultra-sonic intensity at a number of frequencies in the region of 5 megacycles per second. Using light of wave-length $589 \mathrm{m \mu}$, path lengths of about $2.5 \mathrm{~cm}$. and ultra-sonic intensities ranging from zero to about 0.2 watts per $\mathrm{cm}^{2}$, it is found that the envelopes of the diffraction patterns as predicted by the Raman-Nath theory are in excellent agreement with the observations for light scattered in both progressive and standing wave fields, the intensities of positive and negative orders from zero to five being measured.

In view of this close agreement, it appears some. what strange that further experimental evidence should indicate the fundamental assumption of Raman and Nath to be in error. The explanation may possibly be found in the deviations of a practical sound field from the ideal case postulated by these authors, who for simplicity assume the sound field to consist of a rectangular prism of plane waves, the sound intensity over any plane normal to the direction of motion of the waves being constant at any instant. A light ray incident normally on such a field would suffer retardations in phase only. In the practical case, however, the sound field does not fulfil these conditions, particularly at the edges. It is probable that a light ray incident in a direction normal to the axis of the field may suffer some refraction on entering the sound field. Once bent, the ray will be traversing layers of varying refractive index and will suffer some changes in amplitude. Eventually, the various rays may come to a series of foci, thus producing the fringes observed by Bär. In such a field the Raman-Nath theory for oblique incidence might also fail, as noted by Bär.
The fact that the discrepancies between theory and observation are less at the lower frequencies tends to support the above hypothesis since, as the sound wave-length is increased, the probability of a light ray entering the field under the conditions postulated by Raman and Nath is considerably greater. In the case of normal incidence, it is quite conceivable that small changes in amplitude, coupled with the normal changes in phase, give rise to an emergent light wave surface which approximates very closely to that which would be produced if changes in phase alone had occurred. That this is the case appears to be substantiated by the very close agreement between the predicted intensities in the various orders and those measured quantitatively by me and observed qualitatively by Bär.

Until a theory is developed which takes complete account of the nature of a practical sound field, it would hence appear that the theory of Raman and Nath, though based on an assumption which is somewhat erroneous in the practical case, describes most accurately the nature of the diffraction effects observed.

\section{National Research Laboratories, Ottawa. July 11.

\footnotetext{
${ }^{1}$ R. Lucas, C.R., 202, 1165 (March 30, 1936).
} \\ 2 R. Bär, Helv. Phys. Acta, 9, 265 (1936).} F. H. SANDERS.

\section{Absorption Spectrum of Heavy Methane $\left(\mathrm{CH}_{3} \mathrm{D}\right)$ in} the Photographic Infra-Red

WE have recently prepared 40 litres of monodeuteromethane and examined its absorption spectrum in the photographic infra-red from $12,000 \mathrm{~A}$. to $6000 \mathrm{~A}$. under the high dispersion of a grating spectrograph. Examination of our plates reveals complete absence of even the strongest lines of ordinary methane; absence of the heavier methanes was shown by density determinations carried out on a specially fractionated sample.

The spectrum includes a region of intense absorption extending from $8350 \mathrm{~cm} .^{-1}$ to $9000 \mathrm{~cm} .^{-1}$ with a particularly strong and broad maximum at $8617 \mathrm{~cm}^{-1}$. The structure here is complex, but if anything simpler than with ordinary methane, although no obvious regularities stand out. Of particular interest is the occurrence at $9065 \mathrm{~cm}^{-1}$ of a band with a simple structure of regularly spaced lines (approximate spacing $7.5 \mathrm{~cm}^{-1}$ ) and with what may be a $Q$ branch at $9021 \mathrm{~cm}^{-1}$. In the case of methane ${ }^{1}$, a similar band is found at $9047 \mathrm{~cm}^{-1}$ with a spacing of $10.5 \mathrm{~cm} .^{-1}$, and apparently similar bands are also shown by methyl halides ${ }^{2}$ round about $9025 \mathrm{~cm}^{-1}$. The region from $9600 \mathrm{~cm}^{-1}$ to $10,200 \mathrm{~cm}^{-1}$ is occupied by some ten fainter bands. Then from $10,960 \mathrm{~cm}^{-1}$ to $11,220 \mathrm{~cm}^{-1}$ come four bands, that at $11,220 \mathrm{~cm} .^{-1}$ being relatively strong and very similar in appearance to the methane band of the same frequency.

A detailed discussion of this spectrum will shortly be published elsewhere.

W. H. J. CHImDs.

Davy Faraday Laboratory,

H. A. JAHN.

Royal Institution, London, W.1. July 22.

${ }^{1}$ W. H. J. Childs, Proc. Roy. Soc., A, 153, 555 (1936).
${ }^{2}$ H. Verleger, Z. Phys., 98, 342 (1936). 